\title{
On a Cyclic Variation of the Hemispheric Helicity Rule.
}

\author{
A. A. Pevtsov
}

National Solar Observatory, Sunspot, NM 88349, U.S.A.

M. J. Hagyard

Space Science Department, NASA MSFC, AL 35812, U.S.A.

\section{Z. Blehm}

Montana State University, Bozeman, MT 59717, U.S.A.

\author{
J. E. Smith
}

Space Science Department, NASA MSFC, AL 35812, U.S.A.

R. C. Canfield

Montana State University, Bozeman, MT 59717, U.S.A.

T. Sakurai

National Astronomical Observatory, 2-21-1 Ohsawa, Mitaka-shi, Tokyo 181-8588, Japan

\section{Hagino}

Meisei University, 2-590 Nagabuchi, Ohme-shi, Tokyo 198-8655, Japan

\begin{abstract}
.
We report the result of a study of magnetic helicity in solar active regions during 1980-2000. Using the vector magnetograms from four instruments (Haleakala Stokes Polarimeter, Marshall Space Flight Center, Mitaka Solar Flare Telescope and Okayama Observatory Solar Telescope) we calculated the forcefree parameter $\alpha$ and computed a slope $d \alpha / d \varphi$ as the linear fit of $\alpha$ vs. latitude $\varphi$, using annual subsets of data. The hemispheric helicity rule can be expressed in terms of this slope as $d \alpha / d \varphi<0$. We find that each instrument exhibits change in sign of $d \alpha / d \varphi$ for some years. However, we do not see consistency between different instruments in regards to years disobeying the rule. We show that this inconsistency can be attributed to insufficient numbers of active regions in annual subsets of data. We conclude that the present data sets do not allow to make statistically significant inference about possible cyclic variation of the hemispheric helicity rule.
\end{abstract}

For further discussion see Hagyard et al., 2003, ApJ, submitted. 